\title{
Numerical approximation of the heat transfer between domains separated by thin walls
}

\author{
Ramon Codina ${ }^{1, *, \dagger}$ and Guillaume Houzeaux ${ }^{2, \ddagger}$ \\ ${ }^{1}$ Universitat Politècnica de Catalunya, Jordi Girona 1-3, Edifici C1, 08034 Barcelona, Spain \\ ${ }^{2}$ Barcelona Supercomputing Center, Jordi Girona 29, Edifici Nexus II, 08034 Barcelona, Spain
}

\begin{abstract}
SUMMARY
In this paper, we analyse the numerical approximation of the heat transfer problem between two subdomains that we will consider filled with a fluid and separated by a thin solid wall. First of all, we state the problem in the whole domain with discontinuous physical properties. As an alternative and under certain assumptions on the separating walls, a classical Robin boundary condition between the fluid domains is obtained, thus eliminating the solid wall, and according to which the heat flux is proportional to the temperature difference between the two subdomains. Apart from discussing the relation between both approaches, we consider their numerical approximation, considering different alternatives for the first case, that is, the case in which temperatures are also computed in the solid wall. Copyright (C) 2006 John Wiley \& Sons, Ltd.
\end{abstract}

KEY WORDS: heat transfer; domain decomposition; Robin condition

\section{INTRODUCTION}

In this work, we consider the heat transfer problem between two fluid subdomains separated by a wall that we will consider thin [1], a problem with direct application to the thermal analysis of buildings. To this end, we state and compare three possible alternatives:

- In the limit when the thickness of the separating wall tends to zero and assuming that the heat transfer is purely by conduction and the heat capacity of the wall is negligible, we obtain the classical Robin condition for the heat flux on the wall, seen as boundary of each subdomain. In particular, we obtain the surface convection coefficient in terms

\footnotetext{
*Correspondence to: Ramon Codina, Universitat Politècnica de Catalunya, Jordi Girona 1-3, Edifici C1, 08034 Barcelona, Spain.

${ }^{\dagger}$ E-mail: ramon.codina@upc.edu, Web page: http://www.rmee.upc.edu/homes/codina

‡E-mail: guillaume.houzeaux@bsc.es

Contract/grant sponsor: Spanish Ministerio de Educación y Ciencia
} 
of the thickness of the wall and its conductivity. We also indicate how to show that the problem stated this way is well posed.

- If the heat capacity of the wall cannot be considered small, the previous boundary condition is inappropriate for transient problems. To approximate the heat conduction problem in the interior of the solid wall, we propose a finite difference scheme, both through the thickness of this wall and to integrate in time the associated evolution problem.

- We consider finally the most exact alternative, which consists in solving the complete problem, that is, the fluid and thermal problem in each of the fluid subdomains and the thermal problem in the separating solid wall. These are three coupled problems which in turn can be dealt with using different alternatives from the numerical point of view. First, one could solve the whole domain in a fully coupled monolithic way, considering simply that the physical properties are discontinuous. But it is also possible to use a domain decomposition technique. Whereas at the continuous level, both approaches are completely equivalent, the numerical approximation can yield slightly different answers, since the domain decomposition method requires the numerical evaluation of heat fluxes on the boundaries of the subdomains.

For each of the three alternatives described, we approximate the flow equations using a finite element formulation, and state different domain decomposition strategies in order to allow the uncoupling of the computation of the unknowns on each of the subdomains of analysis. The

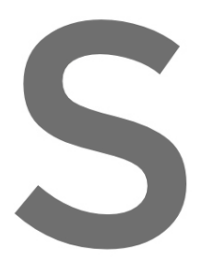
comparison between to estimate the valid options.

The paper is organized as section, which in turn domain, the domain decompostion strategy
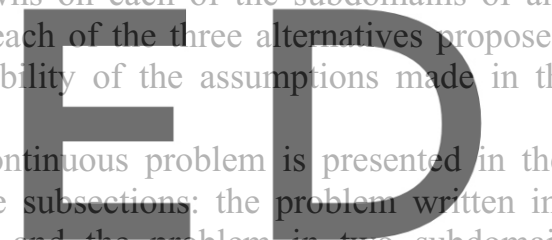

through a Robin condition. For each case, we present the strong and the weak forms of the

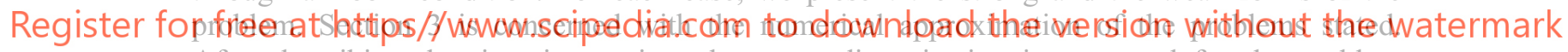
After describing the time integration, the space discretization is presented for the problem written in the whole domain, solved using a domain decomposition strategy and solved in two subdomains coupled through a Robin condition. For the second case, we present a standard discretization of the solid wall as well as a very simple one obtained assuming that the width of this solid is small. Numerical experiments are presented in Section 4 that confirm the interest of this last approach, original from this paper. Finally, conclusions are drawn in Section 5.

\section{CONTINUOUS PROBLEM}

The problem we consider consists in solving the heat equation in a domain $\Omega$ composed by three subdomains: a fluid subdomain that will be called 'subdomain 1', a second fluid subdomain called 'subdomain 2' and a third solid subdomain, that will be called 'subdomain $s$ ', and which will be considered thin (with a dimension at each point much smaller than the others). Due to this geometric feature of subdomain $s$, we will try to simplify the problem. To this end, we will divide this work into three stages.

First, we state the problem in $\Omega$ considering that the physical properties are discontinuous. These properties are density, specific heat and the thermal diffusion (or conduction) coefficient. 

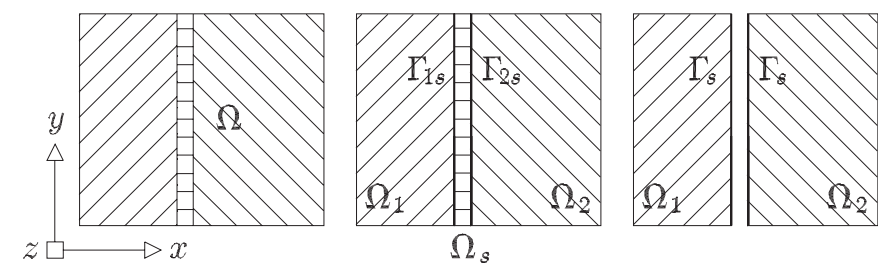

Figure 1. Domain composed by three subdomains. (Left) Whole domain $\Omega$. (Centre) Domain $\Omega$ split into three subdomains. (Right) Domain $\Omega$ approximated by two subdomains.

Since we consider subdomains 1 and 2 filled with fluids, we will also need their viscosity and velocity field, which will be considered known. The situation is illustrated in Figure 1 (1eft).

The second stage consists in dividing the domain $\Omega$ into three subdomains, $\Omega_{1}, \Omega_{2}$ and $\Omega_{s}$. We will present a domain decomposition method that allows to compute the temperature in each domain and accounts for the coupling between them. The situation is illustrated in Figure 1 (centre).

Finally, in the third stage we will profit from the fact the $\Omega_{s}$ is thin. For the sake of conciseness, we will consider $\Omega_{s}$ a flat wall, with the width in the $x$ direction. If this width is small, we will be able to simplify the temperature problem in $\Omega_{s}$ and, in fact, to obtain a domain decomposition problem only involving subdomains $\Omega_{1}$ and $\Omega_{2}$. The sittration is illustrated in Figure adapted. In particula along the normal to normal to the faces

In the continuous
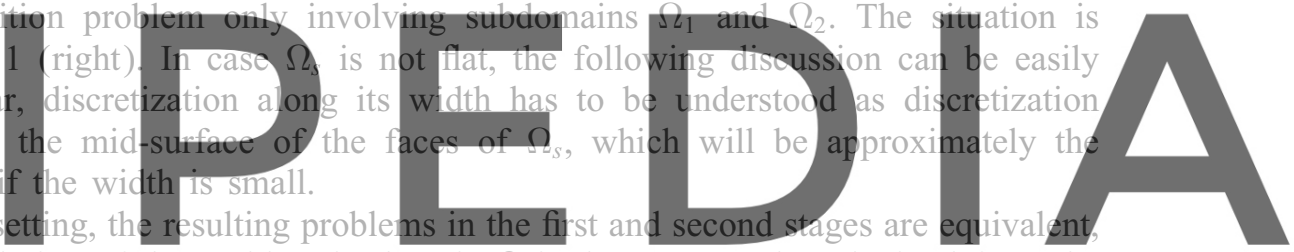

that is to say, the solution of the problem in domain $\Omega$ is the same as that obtained from the

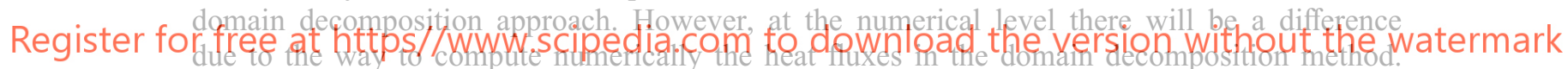
In contrast, the third phase represents an approximation a priori, in the definition of the mathematical model and, more precisely, of the boundary conditions.

\subsection{Problem statement on the whole domain}

2.1.1. Strong form. Let $\Omega$ be an open domain in $\mathbb{R}^{n_{d}}$, where $n_{d}=2$ or 3 is the number of space dimensions, and let $\left(0, t_{\mathrm{f}}\right)$ be the time interval of analysis. Let us consider a known transport velocity $\mathbf{u}$, which we will assume bounded and divergence free, that is to say, $\nabla \cdot \mathbf{u}=0$.

Let $\rho$ be the density, $c_{p}$ the specific heat and $k$ the heat conduction coefficient of the whole medium. These properties will be variable in space and, in fact, discontinuous, since we consider three different materials, one in each subdomain. To simplify the exposition, we will consider these physical properties as piecewise constant.

The equation for the temperature $T$ that governs the heat transfer problem is

$$
\rho c_{p}\left(\partial_{t} T+\mathbf{u} \cdot \nabla T\right)-\nabla \cdot(k \nabla T)=Q \quad \text { in } \Omega \times\left(0, t_{\mathrm{f}}\right)
$$

where $Q$ is a volumetric heat source that we will take constant in time. The solution of this equations requires initial and boundary conditions. Let us split the boundary $\partial \Omega$ of $\Omega$ into 


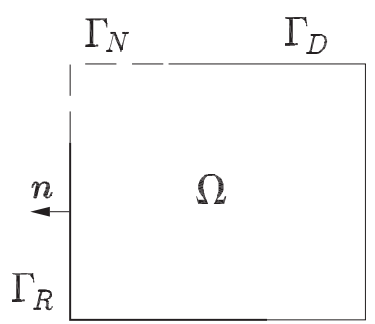

Figure 2. Boundary conditions.

three parts as $\partial \Omega=\Gamma_{\mathrm{D}} \cup \Gamma_{\mathrm{N}} \cup \Gamma_{\mathrm{R}}$, where $\Gamma_{\mathrm{D}}, \Gamma_{\mathrm{N}}$ and $\Gamma_{\mathrm{R}}$ represent the part of the boundary where Dirichlet, Neumann and Robin boundary conditions are prescribed, respectively (see Figure 2). The initial and boundary conditions can thus be written as

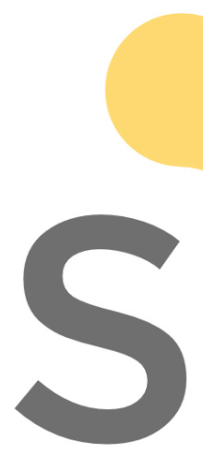

$$
\begin{array}{ll}
T=T_{0} & \text { in } \Omega \times\{0\} \\
T=T_{\mathrm{D}} & \text { on } \Gamma_{\mathrm{D}} \times\left(0, t_{\mathrm{f}}\right)
\end{array}
$$

$$
-k \nabla T \cdot \mathbf{n}=q_{\mathrm{N}} \quad \text { on } \Gamma_{\mathrm{N}} \times\left(0, t_{\mathrm{f}}\right)
$$

In these equations material that occupie material to the exterior the Dirichlet condition
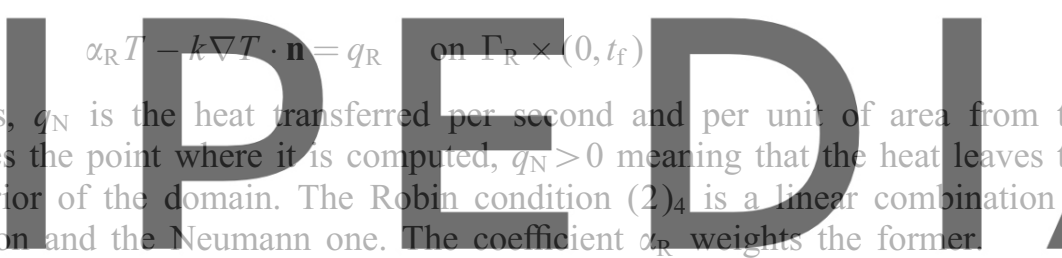

It is well known that this Robin condition can also represent a linearization of a radiation

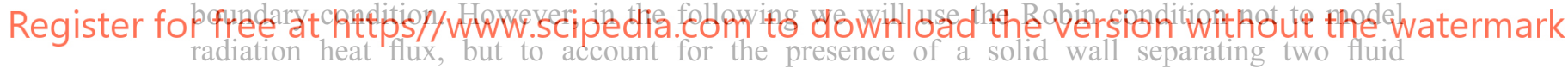
subdomains. The expression of $\alpha_{R}$ in this case is obtained later on.

2.1.2. Weak form. Let us introduce some notation. We start introducing the functional spaces

$$
\begin{aligned}
H^{1}(\Omega):= & \left\{v \in L^{2}(\Omega) \mid \frac{\partial v}{\partial x_{j}} \in L^{2}(\Omega), j=1, \ldots, n_{d}\right\} \\
& V_{\Gamma_{\mathrm{D}}}^{0}:=\left\{v \in H^{1}(\Omega)|v|_{\Gamma_{\mathrm{D}}}=0\right\} \\
& V_{\Gamma_{\mathrm{D}}}^{T_{\mathrm{D}}}:=\left\{v \in H^{1}(\Omega)|v|_{\Gamma_{\mathrm{D}}}=T_{\mathrm{D}}\right\}
\end{aligned}
$$

When $\Gamma_{\mathrm{D}}=\partial \Omega$, we will omit this subscript in the expression of the corresponding functional spaces. The scalar product in $L^{2}(\Omega)$ will be denoted by

$$
(u, v)_{0, \Omega} \equiv(u, v):=\int_{\Omega} u v \mathrm{~d} \Omega
$$

and we will use the notation

$$
\langle f, g\rangle_{\omega}:=\int_{\omega} f g \mathrm{~d} \omega
$$


when the functions $f$ and $g$ are not necessarily square integrable and $\omega$ is either a subdomain of $\Omega$ or part of the boundary $\partial \Omega$. We denote the scalar product in $H^{1}(\Omega)$ by

$$
(u, v)_{1, \Omega}=(u, v)_{0, \Omega}+(\nabla u, \nabla v)_{0, \Omega}
$$

We also define the norms in $L^{2}(\omega)$ and $H^{1}(\omega)$ as

$$
\|u\|_{k, \omega}=\left[(u, u)_{k, \omega}\right]^{1 / 2} \text { for } k=0,1
$$

A weak formulation of the problem is obtained by integrating by parts the diffusive term in Equation (1). Let us introduce the bilinear form

$$
a(T, v):=\left(\rho c_{p} \partial_{t} T, v\right)_{\Omega}+\left(\rho c_{p} \mathbf{u} \cdot \nabla T, v\right)_{\Omega}+(k \nabla T, \nabla v)_{\Omega}-\left\langle\alpha_{\mathrm{R}} T, v\right\rangle_{\Gamma_{\mathrm{R}}}
$$

and the linear form

$$
l(v)=\langle Q, v\rangle_{\Omega}-\left\langle q_{\mathrm{N}}, v\right\rangle_{\Gamma_{\mathrm{N}}}-\left\langle q_{\mathrm{R}}, v\right\rangle_{\Gamma_{\mathrm{R}}}
$$

The weak form of the problem consists in finding $T \in L^{2}\left(0, t_{\mathrm{f}} ; V_{\Gamma_{\mathrm{D}}}^{T_{\mathrm{D}}}\right) \cap L^{\infty}\left(0, t_{\mathrm{f}} ; L^{2}(\Omega)\right)$ such that
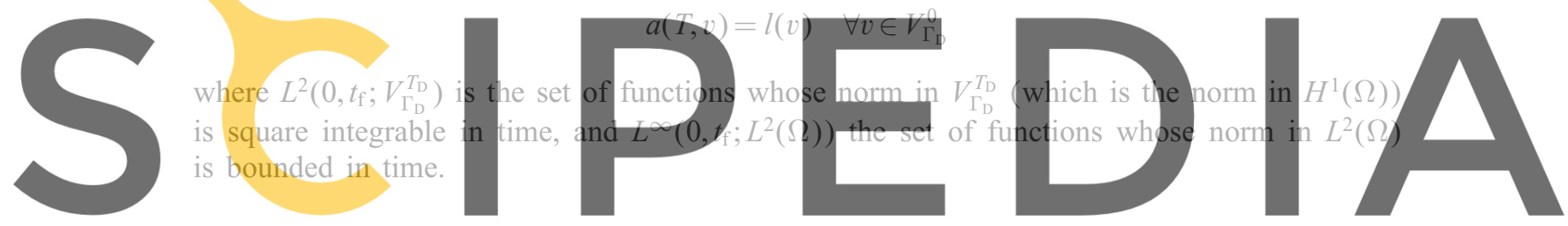

2.2. Domain decomposition with three subdomains

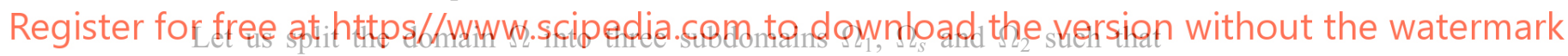

$$
\Omega=\operatorname{int}\left(\overline{\Omega_{1} \cup \Omega_{S} \cup \Omega_{2}}\right)
$$

Let $\Gamma_{1 s}$ be the common interface between $\Omega_{1}$ and $\Omega_{s}$, and $\Gamma_{2 s}$ the common interface between $\Omega_{2}$ and $\Omega_{s}$. In order to simplify the notation, we will only consider homogeneous Dirichlet conditions on the boundary of $\Omega$, that is to say, $T_{D}=0$ and $\Gamma_{N}=\Gamma_{R}=\emptyset$. This domain decomposition is illustrated in Figure 1 (centre). We also define

$$
\Gamma_{\mathrm{D}_{i}}=\Gamma_{\mathrm{D}} \cap \partial \Omega_{i} \quad \text { for } i=1, s, 2
$$

as it is illustrated in Figure 3.

2.2.1. Strong form. The problem written in strong form for each of the three subdomains consists in finding $T_{1}, T_{s}$ and $T_{2}$ such that

$$
\begin{aligned}
\rho_{1} c_{p_{1}}\left(\partial_{t} T_{1}+\mathbf{u}_{1} \cdot \nabla T_{1}\right)-\nabla \cdot\left(k_{1} \nabla T_{1}\right)=Q_{1} & \text { in } \Omega_{1} \times\left(0, t_{\mathrm{f}}\right) \\
\rho_{s} c_{p_{s}} \partial_{t} T_{s}-\nabla \cdot\left(k_{s} \nabla T_{s}\right)=Q_{s} & \text { in } \Omega_{s} \times\left(0, t_{\mathrm{f}}\right) \\
\rho_{2} c_{p_{2}}\left(\partial_{t} T_{2}+\mathbf{u}_{2} \cdot \nabla T_{2}\right)-\nabla \cdot\left(k_{2} \nabla T_{2}\right)=Q_{2} & \text { in } \Omega_{2} \times\left(0, t_{\mathrm{f}}\right)
\end{aligned}
$$




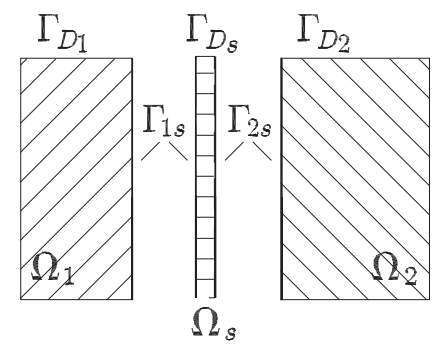

Figure 3. Boundary conditions for the three subdomains.

\section{with the following initial and boundary conditions:}

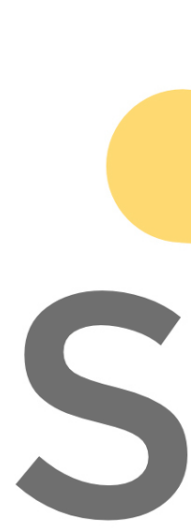

$$
\begin{array}{ll}
T_{1}=\left.T_{0}\right|_{\Omega_{1}} & \text { in } \Omega_{1} \times\{0\} \\
T_{s}=\left.T_{0}\right|_{\Omega_{s}} & \text { in } \Omega_{s} \times\{0\} \\
T_{2}=\left.T_{0}\right|_{\Omega_{2}} & \text { in } \Omega_{2} \times\{0\}
\end{array}
$$

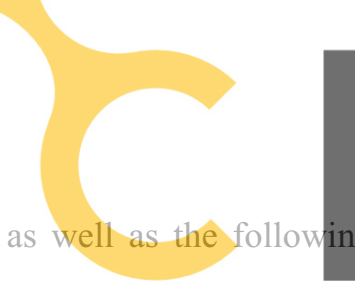

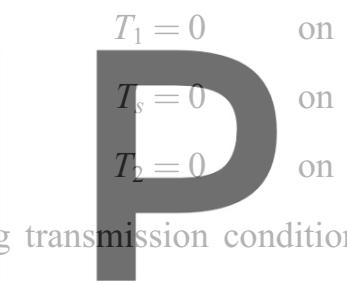

$T_{1}=T_{s}$

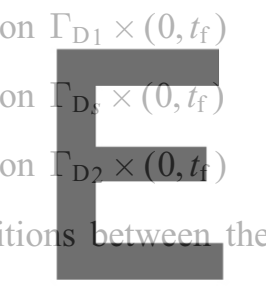

on $\Gamma_{1 s} \times\left(0, t_{\mathrm{f}}\right)$
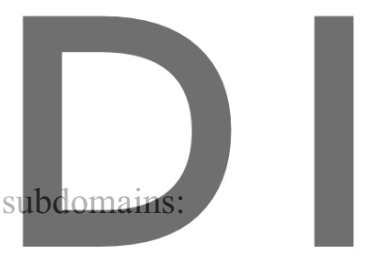

(4)

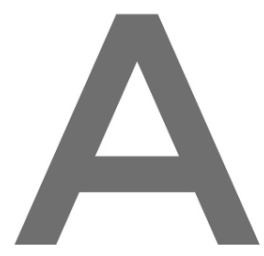

Register for free at https//www.scipedia.c@smițondowintoad (the) version without the watermark

$$
\begin{array}{cc}
T_{2}=T_{s} & \text { on } \Gamma_{2 s} \times\left(0, t_{\mathrm{f}}\right) \\
-k_{2} \nabla T_{2} \cdot \mathbf{n}_{2}=-k_{s} \nabla T_{s} \cdot \mathbf{n}_{2} & \text { on } \Gamma_{2 s} \times\left(0, t_{\mathrm{f}}\right)
\end{array}
$$

which guarantee the continuity of the temperature and the heat flux (which implies conservation of energy) on the interfaces.

2.2.2. Weak form. In practice, the transmission conditions (5) are applied to each subdomain, if there is no overlapping between them, as we consider here. For two adjacent subdomains, such as $\Omega_{1}$ and $\Omega_{s}$, we set the continuity of the heat flux in the former and the continuity of the temperature in the latter. This situation corresponds to what is called a Dirichlet/Neumann coupling [2].

We present next the possibilities of coupling between the three subdomains. Let us define first the bilinear forms $a_{i}$, defined on subdomains $\Omega_{i}$ for $i=1,2, s$, as

$$
a_{i}\left(T_{i}, v\right):=\left(\rho_{i} c_{p_{i}} \partial_{t} T_{i}, v\right)_{\Omega_{i}}+\left(\rho_{i} c_{p_{i}} \mathbf{u}_{i} \cdot \nabla T_{i}, v\right)_{\Omega_{i}}+\left(k_{i} \nabla T_{i}, \nabla v\right)_{\Omega_{i}},-\left\langle\alpha_{\mathrm{R}} T_{i}, v\right\rangle_{\Gamma_{\mathrm{R} i}}
$$

We have included the last term in the bilinear forms in spite of the fact that it is going to be zero for the cases considered. 
The weak form of problem (3)-(5) consists in finding the temperatures

$$
\begin{aligned}
& T_{1} \in L^{2}\left(0, t_{\mathrm{f}} ; V_{\Gamma_{\mathrm{D} 1}}^{0}\right) \cap L^{\infty}\left(0, t_{\mathrm{f}} ; L^{2}\left(\Omega_{1}\right)\right) \\
& T_{s} \in L^{2}\left(0, t_{\mathrm{f}} ; V_{\Gamma_{\mathrm{D}_{s}}}^{0}\right) \cap L^{\infty}\left(0, t_{\mathrm{f}} ; L^{2}\left(\Omega_{s}\right)\right) \\
& T_{2} \in L^{2}\left(0, t_{\mathrm{f}} ; V_{\Gamma_{\mathrm{D}_{2}}}^{0}\right) \cap L^{\infty}\left(0, t_{\mathrm{f}} ; L^{2}\left(\Omega_{2}\right)\right)
\end{aligned}
$$

such that they satisfy the initial conditions $4_{1-3}$ and

$$
\begin{aligned}
a_{1}\left(T_{1}, v_{1}\right) & =\left\langle Q_{1}, v_{1}\right\rangle_{\Omega_{1}}+\left\langle k_{s} \nabla T_{s} \cdot \mathbf{n}_{1}, v_{1}\right\rangle_{\Gamma_{1 s}} & & \forall v_{1} \in V_{\Gamma_{\mathrm{D}_{1}}}^{0} \\
a_{s}\left(T_{s}, v_{s}\right) & =\left\langle Q_{s}, v_{s}\right\rangle_{\Omega_{s}} & & \forall v_{s} \in V_{\Gamma_{\mathrm{D}_{s}}^{0} \cap \Gamma_{1 s} \cup \Gamma_{2 s}} \\
T_{s} & =T_{1} & & \text { on } \Gamma_{1 s} \\
T_{s} & =T_{2} & & \text { on } \Gamma_{2 s} \\
a_{2}\left(T_{2}, v_{2}\right) & =\left\langle Q_{2}, v_{2}\right\rangle_{\Omega_{2}}+\left\langle k_{s} \nabla T_{s} \cdot \mathbb{m}_{2}, v_{2}\right\rangle_{\Gamma_{2 s}} & & \forall v_{2} \in V_{\Gamma_{\mathrm{D} s}}^{0}
\end{aligned}
$$

One can prove that problem (7) and (1) with $T_{\mathrm{D}}=0$ are equivalent (using for example the techniques described in Reference [2]). Note that the continuity of the heat fluxes on $\Gamma_{1 s}$ and $\Gamma_{2 s}$ is alread temperatures is incorpor

2.2.3. Iteration-by-subdomains sulation way to uncouple the calculation of these unknowns consists
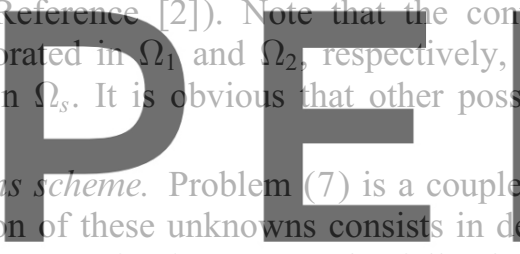

Denoting by $k$ the iteration counter, for $k=1,2, \ldots$ the following uncoupled problems can be Register forlyred for hach timp instant t:

$$
\begin{aligned}
a_{1}\left(T_{1}^{k}, v_{1}\right) & =\left\langle Q_{1}, v_{1}\right\rangle_{\Omega_{1}}+\left\langle k_{s} \nabla T_{s}^{k-1} \cdot \mathbf{n}_{1}, v_{1}\right\rangle_{\Gamma_{1 s} s} & & \forall v_{1} \in V_{\Gamma_{\mathrm{D} 1}}^{0} \\
a_{2}\left(T_{2}^{k}, v_{2}\right) & =\left\langle Q_{2}, v_{2}\right\rangle_{\Omega_{2}}+\left\langle k_{s} \nabla T_{s}^{k-1} \cdot \mathbf{n}_{2}, v_{2}\right\rangle_{\Gamma_{2 s}} & & \forall v_{2} \in V_{\Gamma_{\mathrm{D}_{s}}}^{0} \\
a_{s}\left(T_{s}^{k}, v_{s}\right) & =\left\langle Q_{s}, v_{s}\right\rangle_{\Omega_{s}} & & \forall v_{s} \in V_{\Gamma_{\mathrm{D}_{s}} \cup \Gamma_{1 s} \cup \Gamma_{2 s}} \\
T_{s}^{k} & =T_{1}^{k} & & \text { on } \Gamma_{1 s} \\
T_{s}^{k} & =T_{2}^{k} & & \text { on } \Gamma_{2 s}
\end{aligned}
$$

Obviously, iterations have to be repeated until convergence. This is the so-called iterationby-subdomains method. It is seen that given an initial guess for the temperature at each time instant, $T_{s}^{0}$, the solution of (8) consists in solving the following problems sequentially:

- Equation $(8)_{1}$. The transmission condition is of Neumann type on $\Gamma_{1 s}$ and makes use of the heat flux previously computed in $\Omega_{s}$.

- Equation $(8)_{2}$. The transmission condition is of Neumann type on $\Gamma_{2 s}$ and makes use of the heat flux previously computed in $\Omega_{s}$.

- Equations $(8)_{3-5}$. The transmission conditions are of Dirichlet type and make use of the temperatures just computed in $\Omega_{1}$ and $\Omega_{2}$. 
It is also possible to introduce relaxation factors at the moment of updating the transmission conditions in (8) in order to accelerate (or even to achieve) convergence of the algorithm (see for example References [2-4]).

It is understood in problem (8) that the iterative process is done within each time instant. For a finite difference time discretization as described later on, the iteration has to be performed on the values of the temperature at the time step of consideration. It could be also possible to design schemes in which the temperature at previous iterations is replaced by the temperature at previous time steps, avoiding this way the need to iterate at the expense of relaxing the coupling between subdomains and introducing an explicit operation in the time integration, with the limitations on the time step size that this can imply (see, for example, Reference [5]).

\subsection{Domain decomposition with two subdomains}

In the previous subsection, we have decomposed the original problem into three subproblems, introducing transmission conditions that couple the subdomains by imposing continuity of the temperature and the heat fluxes. In this subsection, we consider a simplified problem, obtained by simplifying the temperature problem in the solid domain $\Omega_{s}$. We will see that, under certain assumptions, it is possible to relate directly the temperature in $\Omega_{1}$ to that in $\Omega_{2}$ through two
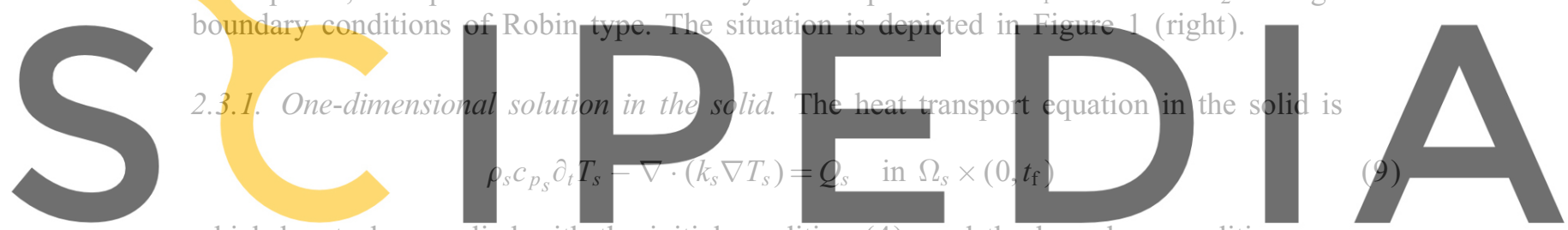

which has to be supplied with the initial condition $(4)_{2}$ and the boundary conditions

$$
T_{s}=T_{2} \text { on } \Gamma_{2 s}
$$

Let us simplify these equations. First, we will consider its dimensionless form. This will allow us to compare its terms. Let us assume that the solid width follows the $x$ direction and its magnitude is $d$, whereas its length (in the $z$ direction) and height (in the $y$ direction) are of order $L$. Let $\Delta T$ be a characteristic temperature difference of the problem, and $\Delta t$ a time scale. Let us introduce the dimensionless variables

$$
T_{s}^{*}=T_{s} / \Delta T, \quad x^{*}=x / d, \quad y^{*}=y / L, \quad z^{*}=z / L, \quad t^{*}=t / \Delta t
$$

We have then that

$$
\frac{\partial T_{s}^{*}}{\partial t^{*}}-\frac{1}{P e_{d}} \frac{\partial^{2} T_{s}^{*}}{\partial x^{* 2}}-\frac{1}{P e_{L}}\left(\frac{\partial^{2} T_{s}^{*}}{\partial y^{* 2}}+\frac{\partial^{2} T_{s}^{*}}{\partial z^{* 2}}\right)=Q_{s} \frac{\Delta t}{\Delta T \rho_{s} c_{p_{S}}}
$$

where

$$
P e_{\ell}=\frac{\rho_{s} c_{p_{s}} \ell^{2}}{k_{s} \Delta t} \quad \text { for } \ell=d \text { and } \ell=L
$$


is a dimensionless number. If we assume that

$$
\begin{aligned}
P e_{d} & \ll 1 \\
d & \ll L
\end{aligned}
$$

then the first and third terms of the left-hand side of (10) are negligible compared to the second, and thus Equation (9) can be approximated by a one-dimensional diffusion equation, which in dimensional form is

$$
-k_{s} \frac{\partial^{2} T_{s}}{\partial x^{2}}=Q_{s} \quad \text { in } \Omega_{s} \times\left(0, t_{\mathrm{f}}\right)
$$

The domain of this equation is illustrated in Figure 4. The boundary conditions for this problem are

$$
\begin{aligned}
& T_{s}=T_{1}\left(x_{1 s}\right) \quad \text { at } x_{1 s} \\
& T_{s}=T_{2}\left(x_{2 s}\right) \quad \text { at } x_{2 s}
\end{aligned}
$$

where we have assumed that $x=x_{1 s}$ and $x_{2 s}$ are the equations of the planes that define the boundaries of the solid. Note that in these last equations both $T /\left(x_{1 s}\right)$ and $T_{2}\left(x_{2 s}\right)$ nay depend on $y$ and $z$.

If we solve the prons
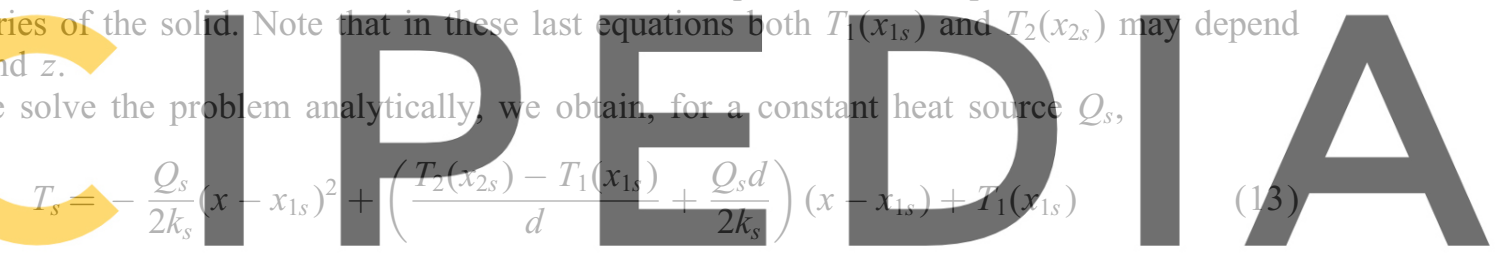

Differentiating this expression we can compute the heat flux at $x_{1 s}$ and $x_{2 s}$ :

Register for free at https//www.scipedia.com to download the version without the watermark

$$
\begin{aligned}
& -\left.k_{s} \frac{\partial T_{s}}{\partial x}\right|_{x_{1 s}}=-\frac{k_{s}}{d}\left[T_{2}\left(x_{2 s}\right)-T_{1}\left(x_{1 s}\right)\right]-\frac{Q_{s} d}{2} \\
& -\left.k_{s} \frac{\partial T_{s}}{\partial x}\right|_{x_{2 s}}=-\frac{k_{s}}{d}\left[T_{2}\left(x_{2 s}\right)-T_{1}\left(x_{1 s}\right)\right]+\frac{Q_{s} d}{2}
\end{aligned}
$$

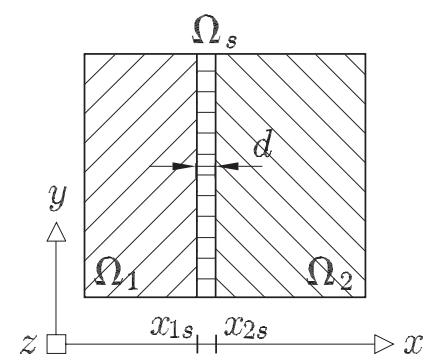

Figure 4. One-dimensional approximation of the problem in $\Omega_{s}$. 
2.3.2. Robin condition at the walls. We have subdomains $\Omega_{1}$ and $\Omega_{2}$ connected through the solid in $\Omega_{s}$, whose temperature is given by the analytical solution we have computed under assumptions (11). This allows us to relate directly the temperature in $\Omega_{1}$ to that of $\Omega_{2}$. We know that at the interface between the solid and the other two subdomains we have, apart from the continuity of the temperature, the continuity of the heat fluxes, expressed by Equation $(5)_{2,4}$. Therefore

$$
\begin{aligned}
& -\left.k_{1} \frac{\partial T_{1}}{\partial x}\right|_{x_{1 s}}=-\frac{k_{s}}{d}\left[T_{2}\left(x_{2 s}\right)-T_{1}\left(x_{1 s}\right)\right]-\frac{Q_{s} d}{2} \\
& -\left.k_{2} \frac{\partial T_{2}}{\partial x}\right|_{x_{2 s}}=-\frac{k_{s}}{d}\left[T_{2}\left(x_{2 s}\right)-T_{1}\left(x_{1 s}\right)\right]+\frac{Q_{s} d}{2}
\end{aligned}
$$

One can check that the heat flux jump is simply the heat source injected between the boundaries of the solid:

$$
-\left.k_{2} \frac{\partial T_{2}}{\partial x}\right|_{x_{2 s}}+\left.k_{1} \frac{\partial T_{1}}{\partial x}\right|_{x_{1 s}}=Q_{s} d
$$

Equations (14) relate explicitly the temperature between subdomains 1 and 2. We can now assume that the solid is very thin, so that planes $x=x_{1 s}$ and $x_{2}$ can be approximated by a
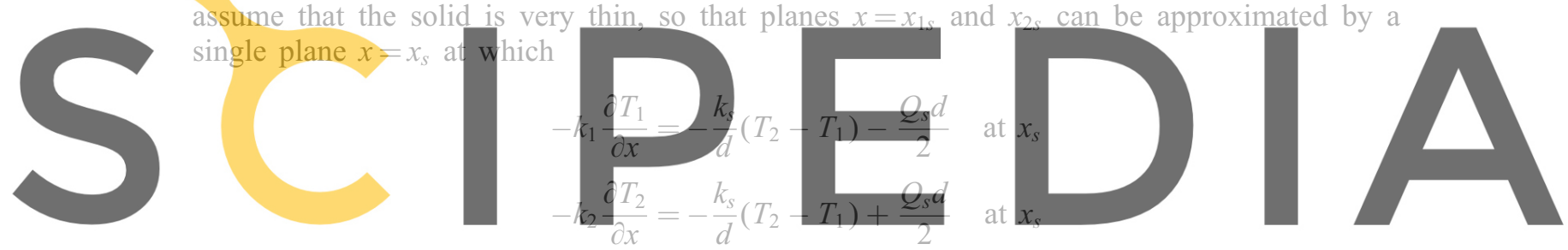

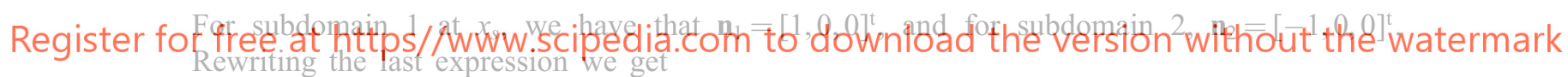

$$
\begin{aligned}
& \alpha_{\mathrm{R}} T_{1}-k_{1} \nabla T_{1} \cdot \mathbf{n}_{1}=\alpha_{\mathrm{R}} T_{2}-\frac{Q_{s} d}{2} \text { at } x_{s} \\
& \alpha_{\mathrm{R}} T_{2}-k_{2} \nabla T_{2} \cdot \mathbf{n}_{2}=\alpha_{\mathrm{R}} T_{1}-\frac{Q_{s} d}{2} \text { at } x_{s}
\end{aligned}
$$

These two conditions are of Robin type and relate subdomains 1 and 2 . The coefficient $\alpha_{R}$ is given by

$$
\alpha_{\mathrm{R}}=-\frac{k_{s}}{d}<0
$$

2.3.3. Strong form. As we did in the case with three subdomains to simplify the analysis, let us assume that the temperature is zero on the boundary of the whole domain $\Omega$. The coupled problem in $\Omega_{1}$ and $\Omega_{2}$ consists in finding $T_{1}$ and $T_{2}$ such that

$$
\begin{array}{ll}
\rho_{1} c_{p_{1}}\left(\partial_{t} T_{1}+\mathbf{u}_{1} \cdot \nabla T_{1}\right)-\nabla \cdot\left(k_{1} \nabla T_{1}\right)=Q_{1} & \text { in } \Omega_{1} \times\left(0, t_{\mathrm{f}}\right) \\
\rho_{2} c_{p_{2}}\left(\partial_{t} T_{2}+\mathbf{u}_{2} \cdot \nabla T_{2}\right)-\nabla \cdot\left(k_{2} \nabla T_{2}\right)=Q_{2} & \text { in } \Omega_{2} \times\left(0, t_{\mathrm{f}}\right)
\end{array}
$$


with the following initial and boundary conditions:

$$
\begin{array}{ll}
T_{1}=\left.T_{0}\right|_{\Omega_{1}} & \text { in } \Omega_{1} \times\{0\} \\
T_{2}=\left.T_{0}\right|_{\Omega_{2}} & \text { in } \Omega_{2} \times\{0\} \\
T_{1}=0 & \text { on } \Gamma_{\mathrm{D}_{1}} \times\left(0, t_{\mathrm{f}}\right) \\
T_{2}=0 & \text { on } \Gamma_{\mathrm{D}_{2}} \times\left(0, t_{\mathrm{f}}\right)
\end{array}
$$

as well as the transmission conditions:

$$
\begin{array}{ll}
\alpha_{\mathrm{R}} T_{1}-k_{1} \nabla T_{1} \cdot \mathbf{n}_{1}=\alpha_{\mathrm{R}} T_{2}-\frac{Q_{s} d}{2} & \text { on } \Gamma_{s} \\
\alpha_{\mathrm{R}} T_{2}-k_{2} \nabla T_{2} \cdot \mathbf{n}_{2}=\alpha_{\mathrm{R}} T_{1}-\frac{Q_{s} d}{2} & \text { on } \Gamma_{s}
\end{array}
$$

which are of Robin type.

2.3.4. Weak form. As before, we consider a homogeneous Dirichlet condition for the temperature on the boundary of the whole domain, and thus on the boundary of each subdomain except on the interface $\Gamma_{s}$, where we have a Robin-type condition, that is, with the notation introduced before,

$$
\Gamma_{\mathrm{R}_{i}}=\Gamma_{s}
$$

Recalling the definition of the bilinear forms $a_{i}$ given by Equation (6), we have that

$$
a_{i}\left(T_{i}, v\right)=\left(\rho_{i} c_{p_{i}} \partial_{t} T_{i}, v\right)_{\Omega_{i}}+\left(\rho_{i} c_{p_{i}} \mathbf{u}_{i} \cdot \nabla T_{i}, v\right)_{\Omega_{i}}+\left(k_{i} \nabla T_{i}, \nabla v\right)_{\Omega_{i}}-\left\langle\alpha_{\mathrm{R}} T_{i}, v\right\rangle_{\Gamma_{s}}
$$

where in this case $\alpha_{R}$ is given by Equation (15).

The weak form of the coupled problem consists in finding $T_{1}$ and $T_{2}$ in the functional spaces introduced earlier, and such that they satisfy the initial conditions $(16)_{1,2}$ and

$$
\begin{array}{ll}
a_{1}\left(T_{1}, v_{1}\right)+\left\langle\alpha_{\mathrm{R}} T_{2}, v_{1}\right\rangle_{\Gamma_{s}}=\left\langle Q_{1}, v_{1}\right\rangle_{\Omega_{1}}+\left\langle Q_{s} d / 2, v_{1}\right\rangle_{\Gamma_{s}} & \forall v_{1} \in V_{\Gamma_{\mathrm{D} 1}}^{0} \\
a_{2}\left(T_{2}, v_{2}\right)+\left\langle\alpha_{\mathrm{R}} T_{1}, v_{2}\right\rangle_{\Gamma_{s}}=\left\langle Q_{2}, v_{2}\right\rangle_{\Omega_{2}}+\left\langle Q_{s} d / 2, v_{2}\right\rangle_{\Gamma_{s}} \quad \forall v_{2} \in V_{\Gamma_{\mathrm{D} 2}}^{0}
\end{array}
$$

2.3.5. Existence and unicity. Let us comment now on the well posedness of the previous coupled problem. To simplify the discussion, let us consider the problem stationary, that is, without the temporal derivative in the bilinear forms that define the problem. In compact form, this problem can be written in the following way: find $\left(T_{1}, T_{2}\right) \in V_{\Gamma_{\mathrm{D} 1}}^{0} \times V_{\Gamma_{\mathrm{D} 2}}^{0}$ such that

$$
a_{12}\left(T_{1}, T_{2} ; v_{1}, v_{2}\right)=l_{12}\left(v_{1}, v_{2}\right) \quad \forall\left(v_{1}, v_{2}\right) \in V_{\Gamma_{\mathrm{D} 1}}^{0} \times V_{\Gamma_{\mathrm{D} 2}}^{0}
$$

where we have defined the bilinear form $a_{12}$ and the linear form $l_{12}$ as

$$
\begin{aligned}
a_{12}\left(T_{1}, T_{2} ; v_{1}, v_{2}\right)= & a_{1}\left(T_{1}, v_{1}\right)+\left\langle\alpha_{\mathrm{R}} T_{2}, v_{1}\right\rangle_{\Gamma_{s}}+a_{2}\left(T_{2}, v_{2}\right)+\left\langle\alpha_{\mathrm{R}} T_{1}, v_{2}\right\rangle_{\Gamma_{s}} \\
= & \left(k_{1} \nabla T_{1}, \nabla v_{1}\right)_{\Omega_{1}}+\left(\rho_{1} c_{p_{1}} \mathbf{u}_{1} \cdot \nabla T_{1}, v_{1}\right)_{\Omega_{1}}+\left\langle\alpha_{\mathrm{R}}\left(T_{2}-T_{1}\right), v_{1}\right\rangle_{\Gamma_{s}} \\
& +\left(k_{2} \nabla T_{2}, \nabla v_{2}\right)_{\Omega_{2}}+\left(\rho_{2} c_{p_{2}} \mathbf{u}_{2} \cdot \nabla T_{2}, v_{2}\right)_{\Omega_{2}}+\left\langle\alpha_{\mathrm{R}}\left(T_{1}-T_{2}\right), v_{2}\right\rangle_{\Gamma_{s}} \\
l_{12}\left(v_{1}, v_{2}\right)= & \left\langle Q_{1}, v_{1}\right\rangle_{\Omega_{1}}+\left\langle Q_{s} d / 2, v_{1}\right\rangle_{\Gamma_{s}}+\left\langle Q_{2}, v_{2}\right\rangle_{\Omega_{2}}+\left\langle Q_{s} d / 2, v_{2}\right\rangle_{\Gamma_{s}}
\end{aligned}
$$


According to Lax-Milgram lemma, problem (18) has a unique solution if the bilinear form $a_{12}$ is both continuous and coercive, that is to say, there exist positive constants $M$ and $N$ such that

$$
\begin{gathered}
\left|a_{12}\left(v_{1}, v_{2} ; w_{1}, w_{2}\right)\right| \leqslant M\left(\left\|v_{1}\right\|_{1, \Omega_{1}}+\left\|v_{2}\right\|_{1, \Omega_{2}}\right)\left(\left\|w_{1}\right\|_{1, \Omega_{1}}+\left\|w_{2}\right\|_{1, \Omega_{2}}\right) \\
a_{12}\left(v_{1}, v_{2} ; v_{1}, v_{2}\right) \geqslant N\left(\left\|v_{1}\right\|_{1, \Omega_{1}}^{2}+\left\|v_{2}\right\|_{1, \Omega_{2}}^{2}\right)
\end{gathered}
$$

for all $\left(v_{1}, v_{2}\right) \in V_{\Gamma_{\mathrm{D} 1}}^{0} \times V_{\Gamma_{\mathrm{D} 2}}^{0}$ and $\left(w_{1}, w_{2}\right) \in V_{\Gamma_{\mathrm{D} 1}}^{0} \times V_{\Gamma_{\mathrm{D} 2}}^{0}$.

Continuity is obtained using standard trace inequalities and using the boundedness assumed for the advection velocity. Coercivity is obtained using the fact the $\alpha_{R}$ is negative and the Poincaré-Friedrichs inequality. Details are omitted (see References [2,6]).

\section{DISCRETE PROBLEM}

\subsection{Time discretization}

The time discretization will be performed using the generalized trapezoidal rule, that is to say, a finite difference scheme. The fully discrete problem will be obtained by discretizing in space the time discrete problem using a finite element formulation. Obviously, it is also possible to start by discretizing first in space and after this in time the resulting initial value problem (method of lines). Nevertheless, we will use the first option. Let us introduce the following notation:

$$
\begin{gathered}
n_{\theta}:=n-1+\theta \\
f^{n_{\theta}}:=\theta f^{n}+(1-\theta) f^{n-1} \\
\delta t:=t^{n}-t^{n-1}
\end{gathered}
$$

where $0<\theta \leqslant 1$. For $\theta=1$ we obtain the backward Euler scheme, of first order, and for $\theta=1 / 2$ the Crank-Nicolson scheme, of second order. Both are unconditionally stable.

The time discretization that we describe next can be applied to any of the problems presented in the previous section. To fix ideas, we present it in the case of the whole domain $\Omega$. Let us define

$$
\begin{aligned}
b^{n_{\theta}}(T, v) & :=\left(\rho c_{p} \mathbf{u}^{n_{\theta}} \cdot \nabla T, v\right)+(k \nabla T, \nabla v)-\left\langle\alpha_{\mathrm{R}} T, v\right\rangle_{\Gamma_{\mathrm{R}}} \\
l^{n_{\theta}}(v) & :=\langle Q, v\rangle_{\Omega}-\left\langle q_{\mathrm{N}}^{n_{\theta}}, v\right\rangle_{\Gamma_{\mathrm{N}}}-\left\langle q_{\mathrm{R}}^{n_{\theta}}, v\right\rangle_{\Gamma_{\mathrm{R}}}
\end{aligned}
$$

The problem discretized in time written in weak form consists in: for $n=1,2, \ldots$, find $T^{n_{\theta}} \in V^{T_{\mathrm{D}}}$ such that

$$
\frac{1}{\theta \delta t}\left(\rho c_{p} T^{n_{\theta}}, v\right)+b^{n_{\theta}}\left(T^{n_{\theta}}, v\right)=\frac{1}{\theta \delta t}\left(\rho c_{p} T^{n-1}, v\right)+l^{n_{\theta}}(v) \quad \forall v \in V^{0}
$$

It is seen from the expression of the space where the solution is sought that we have considered $\Gamma_{\mathrm{D}}=\partial \Omega$. 


\subsection{Space discretization of the whole domain problem}

Let us consider a finite element partition of the whole domain $\Omega$, from which we build up finite element spaces $V_{h}^{T_{\mathrm{D}}} \subset V^{T_{\mathrm{D}}}$ and $V_{h}^{0} \subset V^{0}$ in the usual manner (see, for example, Reference [7]). In all what follows, subscript $h$ will refer to finite element spaces and functions in these spaces.

The Galerkin finite element approximation of problem (20) consists of finding $T_{h}^{n_{\theta}} \in V_{h}^{T_{\mathrm{D}}}$ such that

$$
\frac{1}{\theta \delta t}\left(\rho c_{p} T_{h}^{n_{\theta}}, v_{h}\right)+b^{n_{\theta}}\left(T_{h}^{n_{\theta}}, v_{h}\right)=\frac{1}{\theta \delta t}\left(\rho c_{p} T_{h}^{n-1}, v_{h}\right)+l^{n_{\theta}}\left(v_{h}\right) \quad \forall v_{h} \in V_{h}^{0}
$$

It is well known that this problem displays numerical instabilities when the conduction coefficient is small compared to the advection term, that is, when the flow is convection-dominated. In this case, the numerical solution exhibits oscillations, particularly near boundaries. To overcome this problem, we use a stabilized finite element formulation [8]. However, and since this point is not relevant for what follows and for the sake of simplicity in the exposition, we will restrict ourselves to the Galerkin formulation (21) to approximate problem (20).

The only salient feature of problem (21) is the definition of the physical properties, which are discontinuous when passing from $\Omega_{1}$ to $\Omega_{s}$ and from $\Omega_{s}$ to $\Omega_{2}$. Likewise, we must take into account that at this moment we are considering $\Omega_{s}$ completely discretized by finite elements (even though we consider it a thin domain).

\subsection{Discretization of the domain decomposition problem}

We undertake now the approximation of problem (7), which in practice is implemented in iterative form as indicated in (8). Using the appropriate subscripts for the bilinear form $b$ introduced in Equation (19), the fully discrete problem consists in: for $n=1,2, \ldots$, find $T_{1, h}^{n_{\theta}} \in V_{\Gamma_{\mathrm{D}_{1}, h}}^{0}, T_{s, h}^{n_{\theta}} \in V_{\Gamma_{\mathrm{D}_{s, h}}}^{0}$ and $T_{2, h}^{n_{\theta}} \in V_{\Gamma_{\mathrm{D}_{2}, h}}^{0}$ such that

$$
\begin{aligned}
\frac{\rho_{1} c_{p_{1}}}{\theta \delta t}\left(\delta T_{1, h}^{n_{\theta}}, v_{1, h}\right)_{\Omega_{1}}+b_{1}\left(T_{1, h}^{n_{\theta}}, v_{1, h}\right) & =\left\langle Q_{1}, v_{1, h}\right\rangle_{\Omega_{1}}+\left\langle k_{s} \nabla T_{s, h}^{n_{\theta}} \cdot \mathbf{n}_{1}, v_{1, h}\right\rangle_{\Gamma_{1 s}} \\
\frac{\rho_{s} c_{p_{s}}}{\theta \delta t}\left(\delta T_{s, h}^{n_{\theta}}, v_{s, h}\right)_{\Omega_{s}}+b_{s}\left(T_{s, h}^{n_{\theta}}, v_{s, h}\right) & =\left\langle Q_{s}, v_{s, h}\right\rangle_{\Omega_{s}} \\
T_{s, h}^{n_{\theta}} & =T_{1, h}^{n_{\theta}} \quad \text { on } \Gamma_{1 s} \\
T_{s, h}^{n_{\theta}} & =T_{2, h}^{n_{\theta}} \quad \text { on } \Gamma_{2 s} \\
\frac{\rho_{2} c_{p_{2}}}{\theta \delta t}\left(\delta T_{2, h}^{n_{\theta}}, v_{2, h}\right)_{\Omega_{2}}+b_{2}\left(T_{2, h}^{n_{\theta}}, v_{2, h}\right) & =\left\langle Q_{2}, v_{2, h}\right\rangle_{\Omega_{2}}+\left\langle k_{s} \nabla T_{s, h}^{n_{\theta}} \cdot \mathbf{n}_{2}, v_{2, h}\right\rangle_{\Gamma_{2 s}}
\end{aligned}
$$

for all functions $v_{1, h} \in V_{\Gamma_{\mathrm{D} 1}}^{0}, \quad v_{s, h} \in V_{\Gamma_{\mathrm{D} s} \cup \Gamma_{1 s} \cup \Gamma_{2 s}}^{0}$ and $v_{2, h} \in V_{\Gamma_{\mathrm{D} s}}^{0}$, where $\delta T_{j, h}^{n_{\theta}}:=T_{j, h}^{n_{\theta}}-T_{j, h}^{n-1}$, $j=1, s, 2$. For the continuous problem, the domain decomposition statement gives rise exactly to the same solution as the problem written in the whole domain. However, problems (21) and (22) will have different solutions (apart from the simplification introduced in the boundary conditions) due to the need to approximate the heat fluxes in $(22)_{1,5}$. Note that for the discrete problem in the whole domain, the weak continuity of the heat fluxes is a consequence of disregarding them in the elemental contributions. 
Assuming known the temperatures on $\Gamma_{1, s}$ and $\Gamma_{2, s}$, the problem to be solved for the temperature in the solid domain is $(22)_{2-4}$. These discrete equations are completely general. However, we can simplify them taking into account the geometry of the solid.

3.3.1. Finite element approximation of the solid. This is the most general case. The problem to be solved on the solid is $(22)_{2-4}$. The only remark that needs to be done is the way to compute the heat fluxes that have to be used in Equations $(22)_{1,5}$. In general cases, we can obtain a good approximation to these fluxes by projecting derivatives of the finite element functions onto the space of continuous finite element functions, and with these projections computing the normal derivatives that appear in (22) $)_{1,5}$ (see Reference [9]). However, given the geometry of the solid, we can assume that it is discretized using a structured mesh along its width. This allows us to approximate the heat flux to second order as

$$
\left.\frac{\partial T_{s, h}}{\partial x}\right|_{x_{1 s}}=\frac{1}{2 h}\left(-3 T_{s, 1}+4 T_{s, 2}-T_{s, 3}\right)
$$

where $T_{s, 1}, T_{s, 2}$ and $T_{s, 3}$ are the three first nodes that appear in the solid in the direction normal to the wall, which, as before, has been assumed to be the $x$ direction. In the previous equation, $h$ denotes the distance between nodes. The heat flux at $x_{2 s}$ can be computed in an analogous way.

3.3.2. Finite difference approximation with three points in the solid. The geometry of the solid allows also a simplification that we propose in this paper and whose numerical behaviour is studied in the next section. Assuming as before that the normal to the wall is the $x$ direction, we can assume that there is only one point inside the solid and problem $(7)_{2-4}$ is approximated using finite differences using this single point. Calling $T_{s, h}^{n_{\theta}}$ the temperature at this point assumed to be located at the middle of the solid (for $y$ and $z$ fixed), we have that

$$
\rho_{s} c_{p_{s}} \frac{T_{s, h}^{n_{\theta}}-T_{s, h}^{n}}{\theta \delta t}-k_{s} \frac{\left.T_{1, h}^{n_{\theta}}\right|_{\Gamma_{1 s}}-2 T_{s, h}^{n_{\theta}}+\left.T_{2, h}^{n_{\theta}}\right|_{\Gamma_{2 s}}}{(d / 2)^{2}}=Q_{s}
$$

Using $\theta \delta t$ as time scale, let us introduce the dimensionless number

$$
P e^{\theta}=\frac{\rho_{s} c_{p_{s}} d^{2}}{k_{s} \theta \delta t}
$$

The expression of the temperature at the middle of the solid is

$$
T_{s, h}^{n_{\theta}}=\frac{P e^{\theta} T_{s, h}^{n}-d Q_{s} / \alpha_{\mathrm{R}}+4\left(\left.T_{1, h}^{n_{\theta}}\right|_{\Gamma_{1 s}}+\left.T_{2, h}^{n_{\theta}}\right|_{\Gamma_{2 s}}\right)}{P e^{\theta}+8}
$$

This expression allows us to obtain a temporal approximation to the temperature in the solid wall for the time evolution problem. Note that when $P e^{\theta} \rightarrow 0$ or when the problem reaches a stationary state for which $T_{s, h}^{n_{\theta}}=T_{s, h}^{n}$, the value that follows from (24) is precisely the same that is obtained from (13) at $x=x_{1 s}+d / 2$.

Again in this case we must use the heat fluxes computed at the walls to approximate the problems in $\Omega_{1}$ and $\Omega_{2}$. To this end, we can make use once more of the second-order 
expression (23), which in this particular case becomes

$$
\begin{aligned}
& \left.\frac{\partial T_{s, h}^{n_{\theta}}}{\partial x}\right|_{x_{1 s}}=\frac{1}{d}\left[-\left.3 T_{1, h}^{n_{\theta}}\right|_{\Gamma_{1 s}}+4 T_{s, h}^{n_{\theta}}-\left.T_{2, h}^{n_{\theta}}\right|_{\Gamma_{2 s}}\right] \\
& \left.\frac{\partial T_{s, h}^{n_{\theta}}}{\partial x}\right|_{x_{2 s}}=\frac{1}{d}\left[\left.3 T_{2, h}^{n_{\theta}}\right|_{\Gamma_{2 s}}-4 T_{s, h}^{n_{\theta}}+\left.T_{1, h}^{n_{\theta}}\right|_{\Gamma_{1 s}}\right]
\end{aligned}
$$

Joining $\Gamma_{1 s}$ and $\Gamma_{2 s}$ to define the solid boundary, we can see that the coupled problem consists in finding $T_{1, h}^{n_{\theta}} \in V_{\Gamma_{\mathrm{D}_{1, h}}^{0}}^{0}$ and $T_{2, h}^{n_{\theta}} \in V_{\Gamma_{\mathrm{D}_{2, h}}^{0}}^{0}$ such that

$$
\begin{aligned}
& \frac{\rho_{1} c_{p_{1}}}{\theta \delta t}\left(\delta T_{1, h}^{n_{\theta}}, v_{1, h}\right)_{\Omega_{1}}+b_{1}\left(T_{1, h}^{n_{\theta}}, v_{1, h}\right)-\alpha_{\mathrm{R}_{1}}\left\langle T_{1, h}^{n_{\theta}}, v_{1, h}\right\rangle_{\Gamma_{s}}+\alpha_{\mathrm{R}_{2}}\left\langle T_{2, h}^{n_{\theta}}, v_{1, h}\right\rangle_{\Gamma_{s}} \\
& \quad=\left\langle Q_{1}, v_{1, h}\right\rangle_{\Omega_{1}}+\left\langle Q_{\mathrm{R}}, v_{1, h}\right\rangle_{\Gamma_{s}} \\
& \frac{\rho_{2} c_{p_{2}}}{\theta \delta t}\left(\delta T_{2, h}^{n_{\theta}}, v_{2, h}\right)_{\Omega_{2}}+b_{2}\left(T_{2, h}^{n_{\theta}}, v_{2, h}\right)-\alpha_{\mathrm{R}_{1}}\left\langle T_{2, h}^{n_{\theta}}, v_{2, h}\right\rangle_{\Gamma_{s}}+\alpha_{\mathrm{R}_{2}}\left\langle T_{1, h}^{n_{\theta}}, v_{2, h}\right\rangle_{\Gamma_{s}} \\
& \quad=\left\langle Q_{2}, v_{2, h}\right\rangle_{\Omega_{2}}+\left\langle Q_{\mathrm{R}}, v_{2, h}\right\rangle_{\Gamma_{s}}
\end{aligned}
$$

for all functions $v_{1, h} \in V_{\Gamma_{\mathrm{D}_{1}}}^{0}$ and $v_{2, h} \in V_{\Gamma_{\mathrm{D}_{s}}}^{0}$. We have defined

$$
\begin{gathered}
\alpha_{R_{1}}=\alpha_{\mathrm{R}}\left(1+\frac{2 P e^{\theta}}{P e^{\theta}+8}\right) \\
\alpha_{\mathrm{R}_{2}}=\alpha_{\mathrm{R}}\left(1-\frac{2 P e^{\theta}}{P e^{\theta}+8}\right) \\
Q_{\mathrm{R}}=\frac{4}{P e^{\theta}+8}\left(\alpha_{\mathrm{R}} P e^{\theta} T_{s, h}^{n}-Q_{s} d\right)
\end{gathered}
$$

From the computational point of view, the only difference between this method and the method presented in the next subsection is the expression of these coefficients, that replace $\alpha_{\mathrm{R}}$ and $Q_{s}$. The computational price is to store $T_{s, h}^{n}$. To this end, only an array whose dimension is equal to the number of points inside the solid wall (which is the same as the number of points in the surfaces $\Gamma_{D_{1}}$ and $\Gamma_{D_{2}}$ ) is needed.

\subsection{Discretization of the problem with Robin boundary conditions}

Finally, let us consider the simplest case from the computational point of view and, a priori, the least exact. It consists in the approximation of problem (17) in the domains $\Omega_{1}$ and $\Omega_{2}$ using finite elements and coupling them through a Robin boundary condition. Let us recall that this problem arises from assuming that the temperature in the solid is solution of the stationary one-dimensional heat equation with constant heat source (12). The coupled problem consists in finding $T_{1, h}^{n_{\theta}} \in V_{\Gamma_{\mathrm{D} 1, h}}^{0}$ and $T_{2, h}^{n_{\theta}} \in V_{\Gamma_{\mathrm{D} 2, h}}^{0}$ such that

$$
\begin{aligned}
& \frac{\rho_{1} c_{p_{1}}}{\theta \delta t}\left(\delta T_{1, h}^{n_{\theta}}, v_{1, h}\right)_{\Omega_{1}}+b_{1}\left(T_{1, h}^{n_{\theta}}, v_{1, h}\right)-\alpha_{\mathrm{R}}\left\langle T_{1, h}^{n_{\theta}}-T_{2, h}^{n_{\theta}}, v_{1, h}\right\rangle_{\Gamma_{1 s}} \\
& \quad=\left\langle Q_{1}, v_{1, h}\right\rangle_{\Omega_{1}}+\left\langle Q_{s} d / 2, v_{1, h}\right\rangle_{\Gamma_{s}}
\end{aligned}
$$




$$
\begin{aligned}
& \frac{\rho_{2} c_{p_{2}}}{\theta \delta t}\left(\delta T_{2, h}^{n_{\theta}}, v_{2, h}\right)_{\Omega_{2}}+b_{2}\left(T_{2, h}^{n_{\theta}}, v_{2, h}\right)-\alpha_{\mathrm{R}}\left\langle T_{2, h}^{n_{\theta}}-T_{1, h}^{n_{\theta}}, v_{2, h}\right\rangle_{\Gamma_{2 s}} \\
& \quad=\left\langle Q_{2}, v_{2, h}\right\rangle_{\Omega_{2}}+\left\langle Q_{s} d / 2, v_{2, h}\right\rangle_{\Gamma_{s}}
\end{aligned}
$$

for all functions $v_{1, h} \in V_{\Gamma_{\mathrm{D} 1}}^{0}$ and $v_{2, h} \in V_{\Gamma_{\mathrm{D}_{s}}}^{0}$. Observe that this problem is equivalent to problem (25) taking $P e^{\theta}=0$.

\section{NUMERICAL EXAMPLES}

In this section, we compare the numerical performance of the different approximations introduced in the previous sections. In particular, we have:

- Method 1D: Solution of the whole problem (Section 3.2).

- Method 3D: Solution using domain decomposition discretizing $\Omega_{s}$ with the same finite element partition as for Method 1D. The difference in the converged solution between the results of this and the previous formulation is due only to the computation of the heat fluxes on the walls (Section 3.3.1).

- Method 2D1: Solution using domain decomposition discretizing $\Omega_{s}$ with only three finite difference nodes along its width (Section 3.3.2).

- Method 2Ds: Solution in subdomains $\Omega_{1}$ and $\Omega_{2}$ using a Robin boundary condition between them to simulate the presence of the solid (Section 3.4).

In spite of the fact that the method described as domain decomposition is what we have called Method 3D, methods 2D1 and 2Ds can be also considered as such. Instead of solving in a coupled way for the temperatures in domains $\Omega_{1}$ and $\Omega_{2}$, it is also possible to set an iterative scheme, solving in one of the domains taking as boundary condition the flux computed from the temperature in the other domain. This implementation can be done using a master-slave strategy, as it is described in Reference [9].

\subsection{One-dimensional example}

We consider first a one-dimensional example, whose geometry is shown in Figure 5.

Let us define the thermal diffusion as $\kappa_{i}=k_{i} /\left(\rho_{i} c_{p_{i}}\right)$ for $i=1, s, 2$. The subdomains to be considered have the following data:

$$
\begin{aligned}
& \text { Subdomain 1: Subdomain } s \text { Subdomain 2: } \\
& \Omega_{1}=[0,1] \quad \Omega_{s}=[1,1.1] ; d=0.1 \quad \Omega_{2}=[1.1,2.1] \\
& \kappa_{1}=2.11 \times 10^{-5} \quad \kappa_{s}=3.62 \times 10^{-7}, \times 10^{-6}, \times 10^{-4} \quad \kappa_{2}=2.11 \times 10^{-5}
\end{aligned}
$$

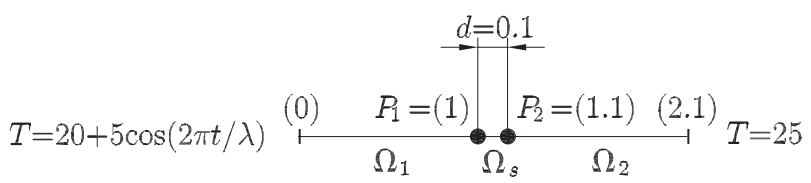

Figure 5. One-dimensional example. Geometry. 
As it is indicated in the properties of the solid in $\Omega_{s}$, we have considered three situations, for $\kappa_{s}=3.62 \times 10^{-7}, 3.62 \times 10^{-6}$ and $3.62 \times 10^{-4}$. From these values we define the dimensionless number

$$
P e_{d}=\frac{d^{2}}{\kappa_{s} \lambda}
$$

so that the three cases considered are

(a) $\kappa_{s}=3.62 \times 10^{-7} \rightarrow P e_{d}=2.76 \times 10^{0}$,

(b) $\kappa_{s}=3.62 \times 10^{-6} \rightarrow P e_{d}=2.76 \times 10^{-1}$,

(c) $\kappa_{s}=3.62 \times 10^{-4} \rightarrow P e_{d}=2.76 \times 10^{-3}$.

The initial and boundary conditions chosen are

$$
\begin{gathered}
T=25 \quad \text { at } t=0 \\
T=20+5 \cos (2 \pi t / \lambda), \quad \lambda=10^{4} \quad \text { at } x=0 \\
T=25 \quad \text { at } x=2.1
\end{gathered}
$$

The time step size has been taken as $\delta t=\lambda / 10$, and the time interval considered $[0,8 \lambda]$. We note that the temperature at $x=0$ oscillates between 15 and 20 in a period $\lambda$. Therefore, we solve eight periods with 10 time steps in each. In the SI unit system, the material in subdomains 1 and 2 is air, whereas the solid has properties similar to those of glass when $\kappa_{s}=3.62 \times 10^{-7}$ (case (a)). The period of oscillation of the boundary condition is then of $2.8 \mathrm{~h}$, approximately.

All the equations have been solved using the finite element method and the backward Euler time integration scheme. For the four methods considered (1D, 3D, 2D1 and 2Ds) we have used a uniform partition of subdomains 1 and 2 with 10 linear elements in each; for method $1 \mathrm{D}$ and method 3D the solid has also been discretized using 10 linear elements of equal length. For method 3D, the derivatives involved in the calculation of the heat flux have been obtained using a second-order scheme, as explained in Section 3.3.1.

Figures 6 (top) show the evolution of the solution for the four methods considered at point $P_{1}$, with abscise $x_{1}=1$, and Figures 6 (centre) the evolution at $P_{2}$, with abscise $x_{2}=1.1$. We can observe that for the three different values of $\kappa_{s}$ considered, method 2D1 gives better results than method $2 \mathrm{D} s$.

Figures 6 (bottom) show the temperature distribution at time $t=3 \times 10^{4}$. It is observed that, in spite of the fact that method 2Ds seems to capture correctly the temperature jump between the walls of the solid, it has a similar error in the two cases considered.

Finally, Figure 7 shows the dependence of the error in terms of dimensionless number $P e_{d}$. This error is computed as

$$
\text { Error } \%=100 \times\left(\frac{\sum_{n=1}^{80} \sum_{i=1}^{2} \sum_{k=1}^{11}\left[\tilde{T}_{i, h}^{n}(k)-T_{i, h}^{n}(k)\right]^{2}}{\sum_{n=1}^{80} \sum_{i=1}^{2} \sum_{k=1}^{11}\left[T_{i, h}^{n}(k)\right]^{2}}\right)^{1 / 2}
$$

$\tilde{T}_{i, h}^{n}(k)$ being the temperature computed with either approximation $3 \mathrm{D}, 2 \mathrm{D} 1$ or $2 \mathrm{Ds}$ at the time level $n$ and at node $k$ of subdomain $i$, whereas $T_{i, h}^{n}(k)$ is the temperature obtained with method 1D, with the same meaning of the subscripts and superscripts. 

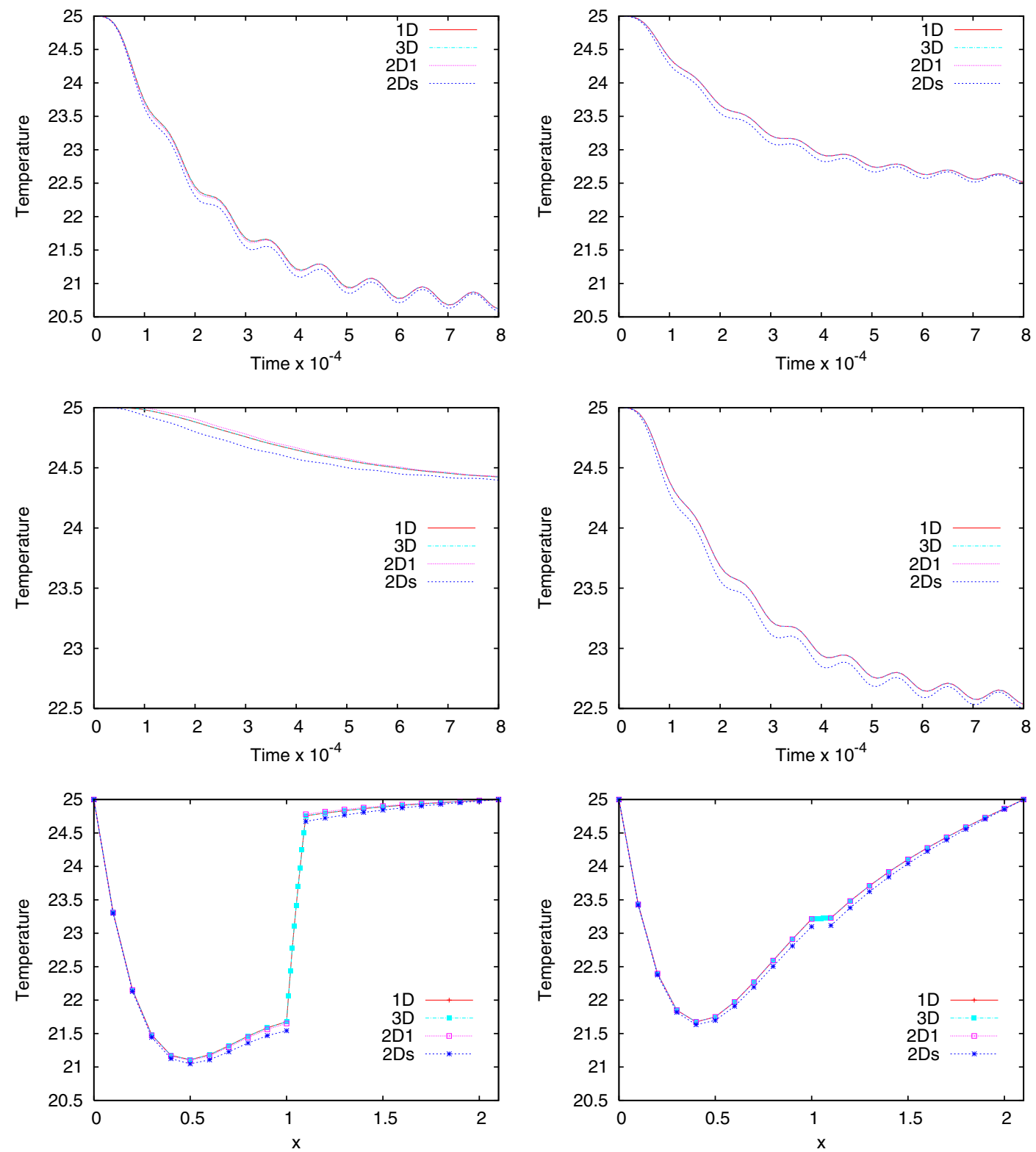

Figure 6. One-dimensional example. (Left) $P e_{d}=2.76 \times 10^{0}$, case (a). (Right) $P e_{d}=2.76 \times 10^{-3}$, case (c). (Top) Evolution of the temperature at $P_{1}$. (Centre) Evolution of the temperature at $P_{2}$.

(Bottom) Temperature distribution at $t=3 \times 10^{4}$.

\subsection{Natural convection in a double cavity}

The goal of this example is to approximate the convective motion of two fluids in two different cavities separated by a thin wall. The geometry of the whole set is shown in Figure 8. 


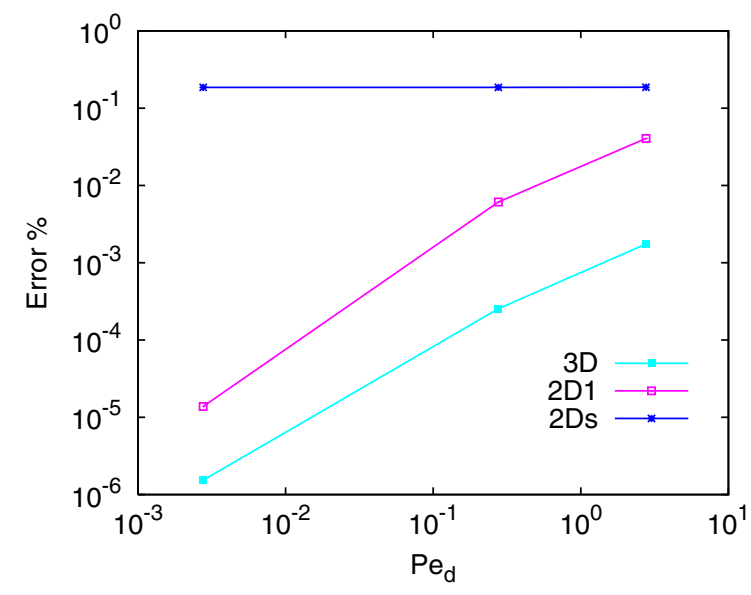

Figure 7. One-dimensional example. Error in terms of $P e_{d}$ (Equation (26)).

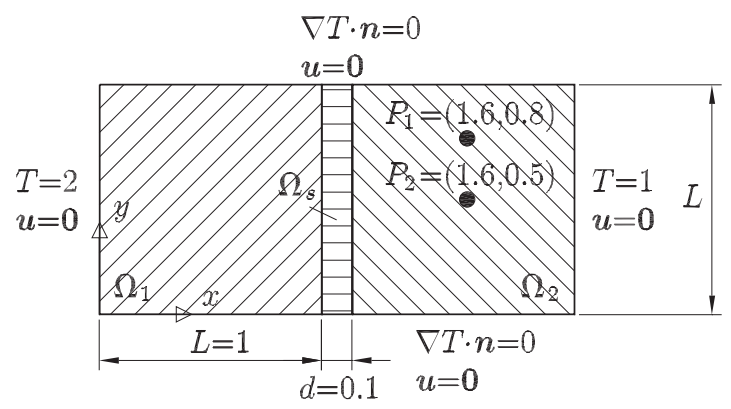

Figure 8. Double cavity. Geometry.

In this case, the heat equation is coupled to the Navier-Stokes equations through the Boussinesq assumption. Let $\mathbf{u}$ be the fluid velocity and $p$ its pressure. We denote the viscosity of each fluid by $\mu_{i}, i=1,2$, and its thermal expansion coefficient by $\beta_{i}, i=1,2$. Let also $\mathbf{g}=(0,-1)$ be the gravity vector. The system of equations that govern this problem is composed by the temperature equation and the Navier-Stokes equations. The former is

$$
\rho c_{p}\left(\partial_{t} T+\mathbf{u} \cdot \nabla T\right)-\nabla \cdot(k \nabla T)=Q \quad \text { in } \Omega \times\left(0, t_{\mathrm{f}}\right)
$$

where $\rho=\rho_{i}, c_{p}=c_{p_{i}}$ and $k_{i}$ are discontinuous, taking values depending on the material $i=1, s, 2$. On the other hand, the Navier-Stokes equations for the velocity and the pressure are

$$
\begin{aligned}
\rho_{1} \partial_{t} \mathbf{u}+\rho_{1}(\mathbf{u} \cdot \nabla) \mathbf{u}-2 \mu_{1} \nabla \cdot \boldsymbol{\varepsilon}(\mathbf{u})+\nabla p & =\rho_{1} \mathbf{g} \beta_{1} T & & \text { in } \Omega_{1} \times\left(0, t_{\mathrm{f}}\right) \\
\nabla \cdot \mathbf{u} & =0 & & \text { in } \Omega_{1} \times\left(0, t_{\mathrm{f}}\right) \\
\mathbf{u} & =\mathbf{0} & & \text { in } \Omega_{s} \times\left(0, t_{\mathrm{f}}\right)
\end{aligned}
$$




$$
\begin{aligned}
\rho_{2} \partial_{t} \mathbf{u}+\rho_{2}(\mathbf{u} \cdot \nabla) \mathbf{u}-2 \mu_{2} \nabla \cdot \boldsymbol{\varepsilon}(\mathbf{u})+\nabla p & =\rho_{2} \mathbf{g} \beta_{2} T & & \text { in } \Omega_{2} \times\left(0, t_{\mathrm{f}}\right) \\
\nabla \cdot \mathbf{u} & =0 & & \text { in } \Omega_{2} \times\left(0, t_{\mathrm{f}}\right)
\end{aligned}
$$

where $\boldsymbol{\varepsilon}(\mathbf{u})$ is the symmetrical part of the velocity gradient. The initial and boundary conditions, illustrated in Figure 8, are

$$
\begin{aligned}
T=0 \quad \text { at } t=0 \\
T=2 \quad \text { at } x=0 \\
T=1 \quad \text { at } x=2.1 \\
\nabla T \cdot \mathbf{n}=0 \quad \text { at } y=0 \\
\nabla T \cdot \mathbf{n}=0 \quad \text { at } y=1 \\
\mathbf{u}=\mathbf{0} \quad \text { at } t=0 \\
\mathbf{u}=\mathbf{0} \quad \text { at } x=0, \quad x=2.1, \quad y=0, y=1
\end{aligned}
$$

The subdomains of this example are characterized by the following data:

$$
\begin{array}{lll}
\text { Subdomain 1: } & \text { Subdomain } s: & \text { Subdomain 2: } \\
\Omega_{1}=[0,1] \times[0,1] & \Omega_{s}=[1,1.1] \times[0,1] & \Omega_{1}=[1.1,2.1] \times[0,1] \\
\rho_{1}=1 & \rho_{s}=1 & \rho_{2}=1 \\
c_{p_{1}}=1 & c_{p_{s}}=1 & c_{p_{2}}=1 \\
k_{1}=1 & k_{s}=10^{-2} & k_{2}=1 \\
\mu_{1}=5 \times 10^{-3} & \mu_{s}=- & \mu_{2}=5 \times 10^{-3} \\
\beta_{1}=125 & \beta_{s}=- & \beta_{2}=125
\end{array}
$$

Let $\Delta T_{i}$, for $i=1,2$, be a characteristic temperature difference of the problems in cavities 1 and 2, respectively. The Prandtl number of the fluids, $P r_{i}$, and the Grashof number of the flows, $G r_{i}$, are defined as

$$
\begin{gathered}
\operatorname{Pr}_{i}=\frac{c_{p_{i}} \mu_{i}}{k_{i}}=5 \times 10^{-3} \\
G r_{i}=\frac{\beta_{i}|\mathbf{g}| \rho_{i}^{2} L^{3} \Delta T_{i}}{\mu_{i}^{2}}=5 \Delta T_{i} \times 10^{6}
\end{gathered}
$$

for $i=1,2$.

Regarding the time integration, the time step size is $\delta t=0.05$ and the time interval $(0,10.0)$.

The flow equations have been discretized in space using a finite element method and in time using the backward Euler time integration scheme. A stabilized formulation has been used 

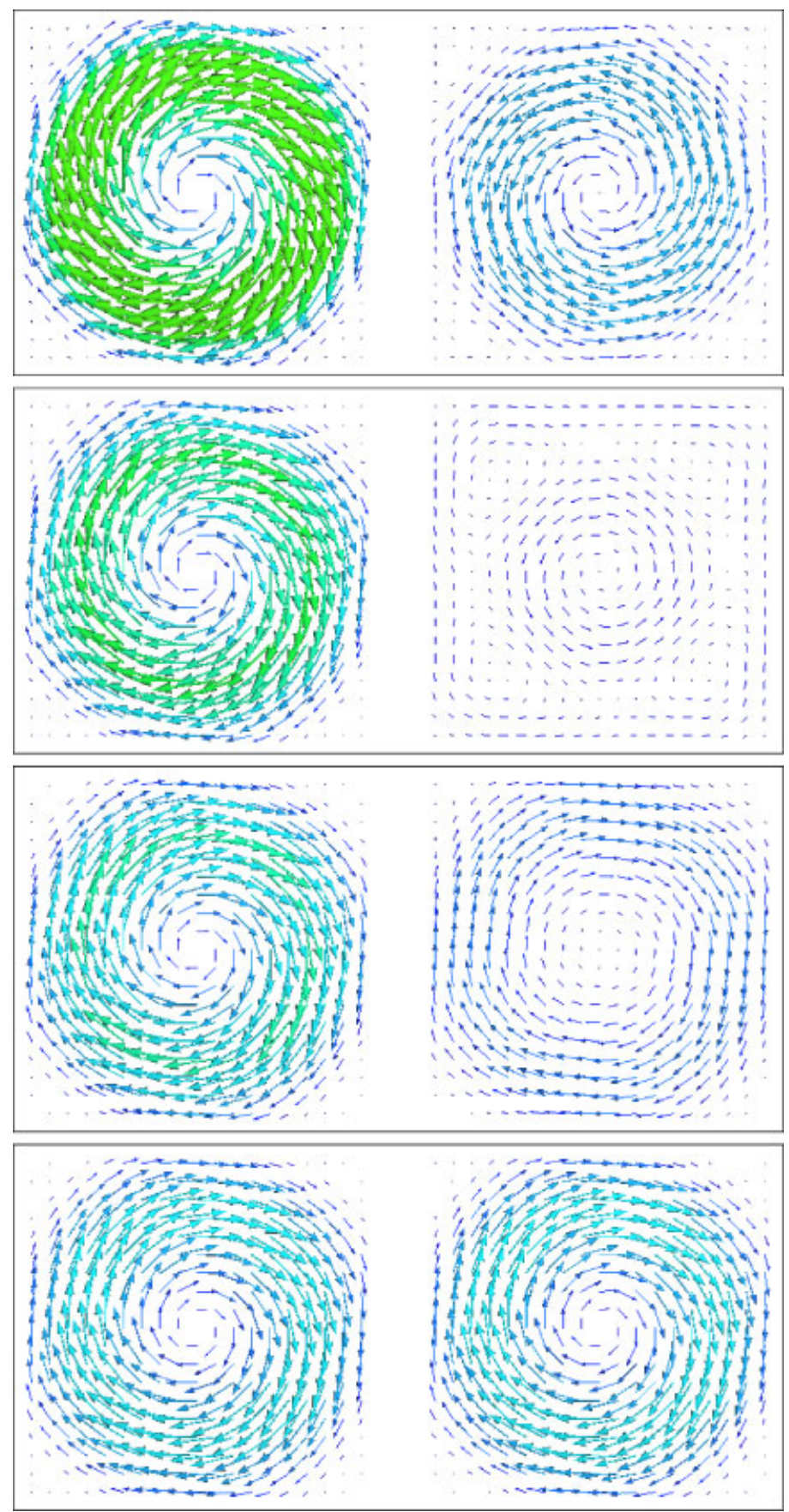

Figure 9. Double cavity. Velocity vectors. From top to bottom: $t=2.0,3.3,5.0,10.0$. 

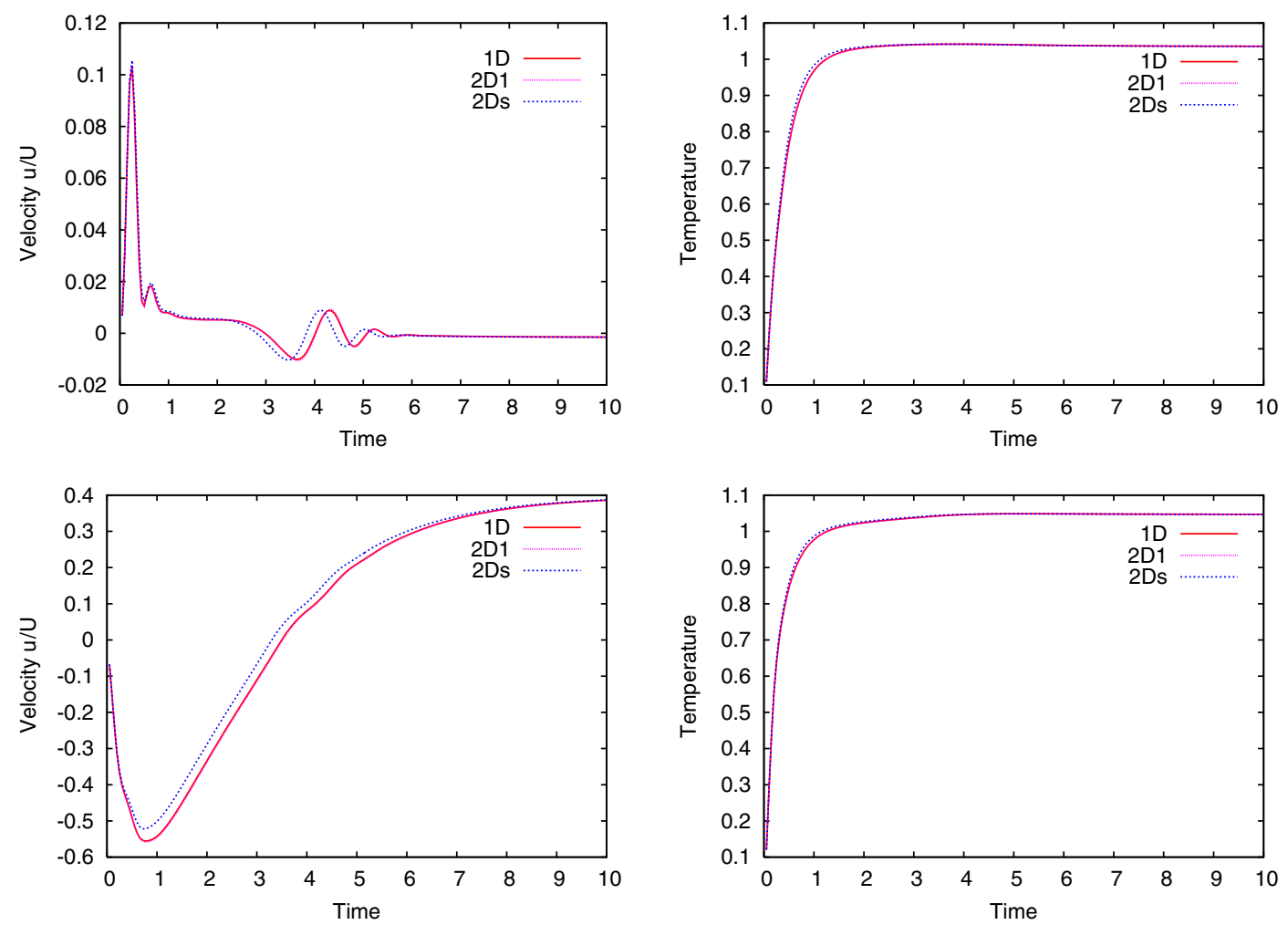

Figure 10. Double cavity. Temporal evolution at two test points. (Left) $x$ velocity normalized by $U=\sqrt{\beta|\mathbf{g}| L \Delta T}$. (Right) Temperature. (Top) Point $P_{2}=(1.6,0.5)$. (Bottom) Point $P_{1}=(1.6,0.8)$.

for both the temperature equation and the Navier-Stokes equations, based on the so-called algebraic subgrid scale (ASGS) formulation presented in Reference [8] following the general ideas proposed in Reference [10].

Our purpose is to compare the results obtained using methods 2D1 and 2Ds with those of the $1 \mathrm{D}$ method. We have used meshes of $20 \times 20$ quadrilateral bilinear elements uniformly distributed in both directions for subdomains 1 and 2; in the 1D method, we have also meshed the solid with 10 bilinear elements uniformly distributed along the width and 20 elements in the vertical direction.

Figure 9 shows the evolution of the velocity vectors in time.

Four stages in the development of the flow can be distinguished:

1. Being zero the initial temperature, the initial Grashof number in subdomain $1, G r_{1}=10^{7}$, doubles that of subdomain 2, which is $G r_{1}=5 \times 10^{6}$. Therefore, the fluid starts rotating faster in cavity 1 . See the first figure from the top in Figure 9.

2. Once the process has started, the subdomains exchange heat through the solid. The Grashof number of both subdomains decrease. Consequently, both flows slow down. See the second figure from the top in Figure 9. 

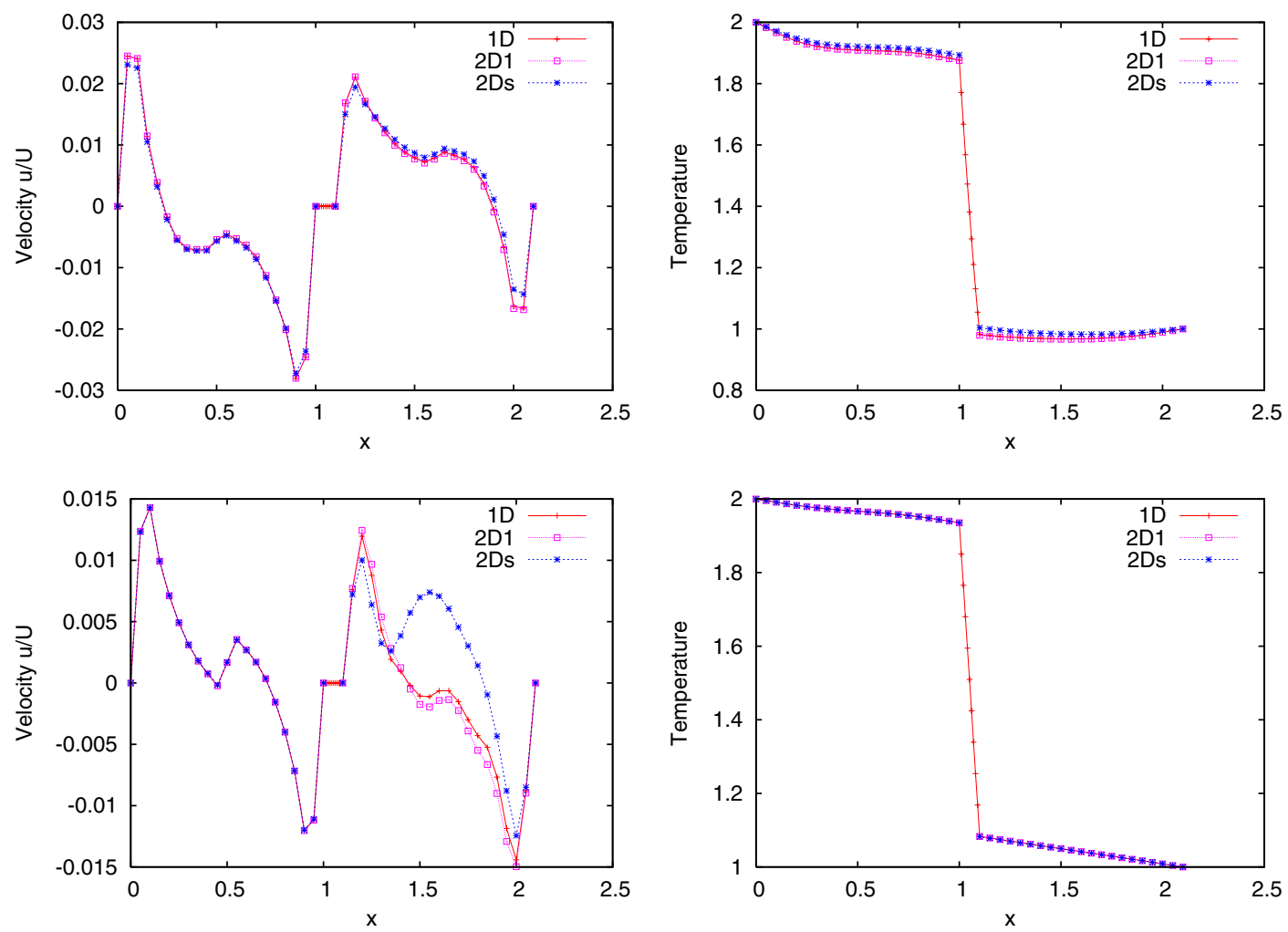

Figure 11. Double cavity. Solution along the vertical cut $y=0.5$. (Left) $x$ velocity normalized by $U=\sqrt{\beta|\mathbf{g}| L \Delta T}$. (Right) Temperature. (Top) Time $t=1$. (Bottom) Time $t=4$.

3. The cavities keep exchanging heat until the value of the Grashof number in subdomain 2 is zero and then the temperature increment changes sign (the hotter wall is $x=1.1$ and not $x=2.1$ ). At this moment, the fluid in cavity 2 inverts its rotating sense. See the third figure from the top in Figure 9.

4. Finally, the systems reaches an equilibrium where the temperature at the middle of the solid is 1.5. At this moment, the fluid rotate in opposite senses with the same intensity, since both fluids have a characteristic temperature difference of 0.5 and the same Grashof number $G r_{1}=G r_{2}=2.5 \times 10^{6}$. See the figure at the bottom of Figure 9 .

In Figure 10, the evolution of the $x$-velocity component and the evolution of the temperature at two points, $P_{1}=(1.6,0.8)$ and $P_{2}=(1.6,0.5)$, are plotted for different methods.

It is observed that the temporal evolution of the temperature is very similar for methods 2D1 and 2Ds. However, method 2D1 allows us to capture much better the temporal evolution of the velocity. The error is almost the $10 \%$ for method $2 \mathrm{D} s$ close to $t=1$. Figure 11 confirms this fact, showing the comparison of the $x$-velocity component and the temperature along the horizontal cut $y=0.5$ for two time steps, corresponding to $t=1$ and to 4 . 


\section{CONCLUSIONS}

In this paper, we have presented several approximations to the heat transfer problem between domains separated by a thin solid wall. We have analysed in particular the basic strategies of numerical approximation of the solid and its modelling through a Robin-type boundary condition. The derivation of this condition is based on the fact that the temperature difference between the interfaces with the two subdomains is produced instantaneously, and thus it is foreseeable that in transient problems this approximation will be poor. The numerical examples presented confirm this fact. The errors that arise with this approximation do not spoil completely the numerical solution, but can be quite significant. To avoid them, we have proposed to approximate the heat flux in the interior of the solid through a finite difference scheme of only three points, one in the middle of the solid along its width and the other two on its boundaries. This approximation has turned out to be a significant improvement, and also very easy to implement with minor changes with respect to the classical formulation with the Robin boundary condition.

\section{ACKNOWLEDGEMENTS}

The research of Dr Houzeaux has been partly done under a Ramon y Cajal contract with the Spanish Ministerio de Educación y Ciencia.

\section{REFERENCES}

1. Incropera FP, Dewitt DP. Fundamentals of Heat and Mass Transfer (4th edn). Wiley: New York, 1996.

2. Quarteroni A, Valli A. Domain Decomposition Methods for Partial Differential Equations. Oxford Science Publications: Oxford, 1999

3. Houzeaux G, Codina R. An overlapping Dirichlet/Robin domain decomposition method. Journal of Computational and Applied Mathematics 2003; 158(2):243-276.

4. Funaro D, Quarteroni A, Zanolli P. An iterative procedure with interface relaxation for domain decomposition methods. SIAM Journal on Numerical Analysis 1988; 25:1213-1236.

5. Felippa CA. Partitioned analysis for coupled mechanical systems. Engineering Computations 1988; 5:123-133.

6. Oden JT, Demkowicz LF. Applied Functional Analysis for Science and Engineering. CRC Press: Boca Raton, FL, 1996.

7. Reddy JN, Gartling DK. The Finite Element Method in Heat Transfer and Fluid Dynamics. CRC Press: Boca Raton, FL, 1994.

8. Codina R. On stabilized finite element methods for linear systems of convection-diffusion-reaction equations. Computer Methods in Applied Mechanics and Engineering 2000; 188:61-82.

9. Houzeaux G, Codina R. A Chimera method based on a Dirichlet/Neumann (Robin) coupling for the NavierStokes equations. Computer Methods in Applied Mechanics and Engineering 2003; 192:3343-3377.

10. Hughes TJR. Multiscale phenomena: Green's function, the Dirichlet-to-Neumann formulation, subgrid scale models, bubbles and the origins of stabilized formulations. Computer Methods in Applied Mechanics and Engineering 1995; 127:387-401. 